\title{
V/Q Scanning Using SPECT and SPECT/CT
}

\author{
Paul J. Roach, Geoffrey P. Schembri, and Dale L. Bailey \\ Department of Nuclear Medicine, Royal North Shore Hospital, and Sydney Medical School, University of Sydney, Sydney, Australia
}

Learning Objectives: On successful completion of this activity, participants should be able to describe (1) advantages and shortcomings of planar versus SPECT V/Q scanning, (2) advantages and disadvantages of CT pulmonary angiography versus V/Q SPECT in the investigation of pulmonary embolism, and (3) an overview of image acquisition, processing, display, and reporting of V/Q SPECT studies.

Financial Disclosure: The authors of this article have indicated no relevant relationships that could be perceived as a real or apparent conflict of interest.

CME Credit: SNMMI is accredited by the Accreditation Council for Continuing Medical Education (ACCME) to sponsor continuing education for physicians. SNMMI designates each JNM continuing education article for a maximum of 2.0 AMA PRA Category 1 Credits. Physicians should claim only credit commensurate with the extent of their participation in the activity. For CE credit, participants can access this activity through the SNMMI Web site (http:// www.snmmi.org/ce_online) through September 2016.

Planar ventilation-perfusion (V/Q) scanning is often used to investigate pulmonary embolism; however, it has well-recognized limitations. SPECT overcomes many of these through its ability to generate 3-dimensional imaging data. V/Q SPECT has higher sensitivity, specificity, and accuracy than planar imaging and a lower indeterminate rate. SPECT allows for new ways to display and analyze data, such as parametric $V / Q$ ratio images. Compared with CT pulmonary angiography, SPECT has higher sensitivity, a lower radiation dose, fewer technically suboptimal studies, and no contrast-related complications. Any nuclear medicine department equipped with a modern hybrid scanner can now perform combined V/Q SPECT with CT (using low-dose protocols) to further enhance diagnostic accuracy. V/Q SPECT (with or without CT) has application in other pulmonary conditions and in research.

Key Words: pulmonary embolism; V/Q scintigraphy; SPECT; SPECT/CT

J Nucl Med 2013; 54:1588-1596

DOI: 10.2967/jnumed.113.124602

$\mathbf{S}$

nce its first description by Wagner et al. in 1964 (1), the planar lung scan has been one of the most commonly performed studies in nuclear medicine. It is, however, a test with significant limitations, which have had an adverse impact on its reputation $(2,3)$. Because planar imaging is a 2-dimensional technique, it has inherent limitations, especially related to overlap of anatomic segments. Assigning defects to specific lung segments is often difficult, and the variability in segment size and shape between patients makes accurate determination of the extent of embolic involvement in individual segments a challenge (2). Embolic defects may not be detected if there is shine-through from underlying lung segments with normal perfusion, thus resulting in an underestimation of the extent of perfusion loss (4). Furthermore, the medial basal segment of the right lower lobe is often not

Received Apr. 30, 2013; revision accepted Jun. 28, 2013.

For correspondence contact: Paul J. Roach, Department of Nuclear Medicine, Royal North Shore Hospital, St. Leonards, NSW, 2065, Australia.

E-mail: proach@nsccahs.health.nsw.gov.au

Published online Aug. 1, 2013.

COPYRIGHT (C) 2013 by the Society of Nuclear Medicine and Molecular Imaging, Inc. visualized on planar scans $(2,5)$. Added to these factors is the widespread use of probabilistic reporting criteria, and a relatively high indeterminate rate, both of which have caused significant dissatisfaction among referring physicians $(6,7)$. It is unsurprising that contrast-enhanced CT pulmonary angiography (CTPA), with its binary reporting approach (i.e., positive or negative), has now become the preferred imaging test to assess pulmonary embolism (PE) in many institutions, particularly in the United States $(8,9)$.

\section{ADVANTAGES OF SPECT OVER PLANAR IMAGING}

Although SPECT is routinely used in many areas of nuclear medicine because of its ability to image in 3 dimensions, lung imaging remains a 2-dimensional technique in many institutions. SPECT imaging of the lungs avoids segmental overlap and shine-through of adjacent lung and can more accurately define the size and location of perfusion defects in individual segments (2).

Studies comparing planar and SPECT lung scanning have consistently demonstrated the superiority of SPECT over planar imaging. Studies on dogs (10) and pigs (11), as well as studies using Monte Carlo simulation (5), have all shown a higher sensitivity for the detection of PE with SPECT than with planar imaging. In humans, Bajc et al. found SPECT to be more sensitive than planar imaging $(100 \%$ vs. $85 \%)$ in the detection of PE (12). SPECT had less interobserver variation and better delineation of mismatched defects than did planar imaging in this study. Collart et al. also demonstrated that SPECT was more specific than planar imaging (96\% vs. $78 \%$ ) and had better reproducibility, both intraobserver (94\% vs. $91 \%$ ) and interobserver (88\% vs. $79 \%$ ) (13). In a study of 83 patients, Reinartz et al. demonstrated that, compared with planar imaging, SPECT had a higher sensitivity (97\% vs. $76 \%$ ), specificity (91\% vs. $85 \%$ ), and accuracy (94\% vs. $81 \%$ ) (14). In this series, SPECT increased the detection of segmental defects by approximately $13 \%$ and subsegmental defects by over $80 \%$. Based on pooled literature, SPECT has sensitivities ranging from $80 \%$ to $100 \%$ and specificities ranging from $93 \%$ to $100 \%$ (8). Ventilation-perfusion (V/Q) SPECT imaging has also been consistently shown to have a much lower indeterminate rate than planar imaging, typically less than $5 \%(8,15-19)$. Leblanc et al. demonstrated that SPECT has a very high negative predictive value $(98.5 \%)$ for PE (20). In that study of 108 


\begin{tabular}{ll}
\hline \multicolumn{1}{c}{ Parameter } & \multicolumn{1}{c}{ Description } \\
\hline SPECT acquisition & $3^{\circ}$ steps over $360^{\circ}$ \\
\hline Acquisition time per projection & $12 \mathrm{~s}$ (ventilation); $8 \mathrm{~s}$ (perfusion) \\
Collimator & Low-energy, high resolution \\
Matrix size & $128 \times 128$ (64 $\times 64$ can also be used) \\
Reconstruction & Ordered-subset expectation maximization (8 iterations, 4 subsets) \\
Postreconstruction filter & 3-dimensional Butterworth; cutoff, 0.8 cycles/cm; order, 9 \\
& \\
\hline *Protocol from Royal North Shore Hospital, Sydney. & \\
\hline
\end{tabular}

patients, only $3 \%$ of studies were considered to be indeterminate for PE.

The literature to date is quite consistent and, taken together, indicates that SPECT has a greater sensitivity and specificity, higher reproducibility, and lower indeterminate rate than planar lung scintigraphy.

\section{V/Q SPECT}

\section{Technique}

As with planar imaging, the usual approach with SPECT is to perform the ventilation study followed by the perfusion study.

For imaging of ventilation, several options exist. Inert radioactive gases such as ${ }^{81 \mathrm{~m}} \mathrm{Kr}$ and ${ }^{133} \mathrm{Xe}$ most accurately represent regional ventilation; however, these are used in only a limited number of centers because of the requirement for continuous administration during the acquisition and the high cost and short shelf-life of the ${ }^{81 \mathrm{~m}} \mathrm{Kr}$ generator (21). Although ${ }^{133} \mathrm{Xe}$ gas has the advantage of a longer half-life, it is a less than ideal choice because of recirculation, low $\gamma$-photon energy, and hence poor spatial resolution (21). Given these limitations, ${ }^{99 \mathrm{~m}} \mathrm{Tc}-$ labeled particulate aerosols such as ${ }^{99 \mathrm{~m}} \mathrm{Tc}$-diethylenetriaminepentaacetic acid ( ${ }^{99 \mathrm{~m}}$ Tc-DTPA) or the ultrafine carbon-labeled nanoparticle ${ }^{99 \mathrm{~m}} \mathrm{Tc}-$ Technegas (Cyclomedica) are much more widely used because of their greater availability, low cost, and good image quality (19). Technegas is an ideal agent for ventilation SPECT because of its small particle size $(30-60 \mathrm{~nm})$, resulting in greater alveolar penetration and less central deposition than a nebulizer-produced aqueous radioaerosol such as ${ }^{99 \mathrm{~m}}$ Tc-DTPA (22). However, in the United States (where Technegas is not commercially available, a factor that has significantly prevented the transition to SPECT (23)), there is little option but to use agents such as ${ }^{99 m}$ Tc-DTPA or ${ }^{99 \mathrm{~m}} \mathrm{Tc}$-sulfur colloid. Although ${ }^{99 \mathrm{~m} T c-D T P A}$ image quality is adequate in many patients, Technegas is clearly superior in patients with obstructive lung disease because of its better peripheral penetration (24). The typical administered dose of $99 \mathrm{mTc}$ based ventilation agents is $30-50 \mathrm{MBq}$, which is comparable to that used in planar imaging $(14,17,20)$.

Perfusion is generally assessed using ${ }^{99 \mathrm{~m} T c-m a c r o a g g r e g a t e d}$ albumin (17). The dose of ${ }^{99 \mathrm{~m}} \mathrm{Tc}$-macroaggregated albumin is dependent on the ventilation agent and dose but is typically on the order of $100-250 \mathrm{MBq}$ if a technetium-based ventilation agent is used. The European Association of Nuclear Medicine guidelines for V/Q SPECT recommend doses at the low end of this range, but ultimately the doses administered should be determined by each institution on the basis of the image quality obtained (which is influenced by factors such as collimator choice, $\gamma$-camera sen- sitivity, and processing parameters) and local radiation protection guidelines (25).

For pregnant patients, the administered dose is usually reduced by half for both the ventilation and the perfusion agents (25), thus requiring a longer acquisition so as to maintain images of good quality. Some centers advocate omitting the ventilation scan; however, the radiation savings from this approach are minimal and diagnostic accuracy may be adversely affected (26).

\section{Image Acquisition, Processing, Display, and Reporting}

Multidetector $\gamma$-cameras are preferred for V/Q SPECT (19). Generally, a total imaging time of $20-30 \mathrm{~min}$ is required to complete both the ventilation and the perfusion SPECT scansless time than is required for traditional 6- or 8-view planar imaging $(14,20)$. Typical acquisition and processing parameters are shown in Table 1 . Images are best reviewed on a workstation using a dedicated software display package that allows automatic or manual image coregistration of ventilation and perfusion data and review of images in each of the orthogonal planes (27).

For those reporting specialists familiar with planar images, these can be generated from SPECT data using several approaches. Although Bailey et al. have described a technique using reprojection (28), many of the commercial vendors offer a simpler approach using an angular summing technique (14). With this approach, images are generated by summing several consecutive projections from the SPECT acquisition. This approach can blur small defects because data are acquired over an arc; however, the images produced approximate true planar images (29). These pseudoplanar images give a familiar and rapid view of the lungs for quick evaluation and may be of particular value during the transition phase from planar imaging to SPECT imaging.

More advanced data processing can be performed with SPECT data. First, defect contrast on perfusion SPECT can be further enhanced by subtracting the background activity remaining from the preceding ventilation scan $(12,30)$. Further, by examining the pixel-based V/Q ratio, quotient images can be generated from SPECT data. These images can facilitate image reporting and improve the demonstration of defect location and extent (Fig. 1) (30). Objective quantification of V/Q ratios is another processing technique that has been shown to increase accuracy and reduce the number of indeterminate studies $(31,32)$.

V/Q studies are usually reported using the European Association of Nuclear Medicine guidelines, which recommend that studies be reported as positive for PE if there is V/Q mismatch of at least 1 segment or 2 subsegments that conforms to the 


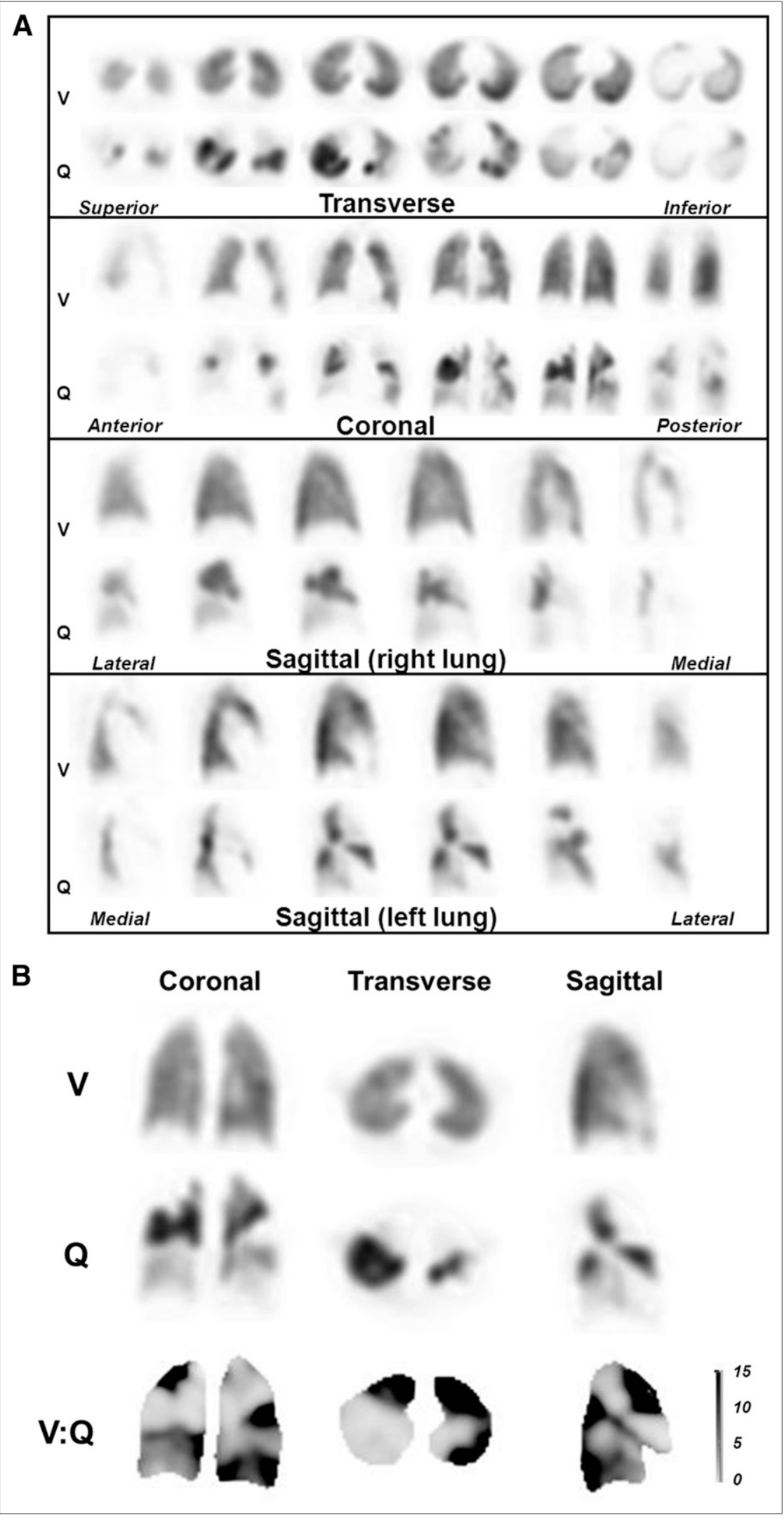

FIGURE 1. (A). Example of patient with multiple bilateral PE. Ventilation and perfusion images show multiple mismatched perfusion defects. (B) Representative ventilation, perfusion, and $\mathrm{V} / \mathrm{Q}$ quotient images. Dark areas on $\mathrm{V} / \mathrm{Q}$ quotient images, denoting high $\mathrm{V} / \mathrm{Q}$ ratio, are indicative of $V / Q$ mismatch. (Reprinted with permission from (19).) pulmonary vascular anatomy (17). Probabilistic reporting as used for planar scanning is not recommended, and has not been validated, for V/Q SPECT $(14,33)$.

\section{Comparison with CTPA}

Multidetector CTPA has evolved to the point where it is frequently used as the primary imaging investigation in patients with potential PE (34). This is certainly the case in the United States, where it has supplanted the V/Q scan as the initial imaging test for the assessment of PE in many institutions $(8,9)$. This preference has happened for many reasons, including better availability in many centers (especially after hours), rapid acquisition time, the ability to diagnose conditions other than PE that could be accounting for the patient's symptoms, and referrer preference for binary reporting $(8,9)$.

There are, however, some significant limitations that can affect the use of CTPA for the investigation of PE. First, several studies have shown that the sensitivity of CTPA is less than desirable $(18,35)$. In the large PIOPED II study (Prospective Investigation of Pulmonary Embolism Diagnosis), the sensitivity of CTPA was $83 \%$ (78\% when technically suboptimal studies were included) (35). Accuracy was particularly suboptimal if there was discordance between the scan results and clinical likelihood (a finding similar to that noted with planar V/Q scintigraphy in the original PIOPED study (36)). Second, technical artifacts can affect image quality. These are primarily related to poor contrast opacification of the pulmonary arteries, motion artifacts, and image noise related to the body habitus of some patients (37). Indeterminate rates due to technical factors have been estimated at between $5 \%$ and $11 \%(38,39)$. In pregnant patients, the rate is even higher, occurring in as many as one third of CTPA procedures, even with 64-slice CT scanners $(40,41)$, and is thought to be attributable to increased pressure in the inferior vena cava during pregnancy. Third, complications can result from the use of intravenous contrast material. In the PIOPED 2 study, 22\% of patients were excluded because of contrast allergy and impaired renal function (35). It has been reported that CTPA is complicated by some type of immediate contrast reaction in 3\% (42) and contrastinduced nephropathy in 1\%-3\% of patients (43). Fourth, radiation exposure can be high from CT. The radiation dose to the 
TABLE 2

Summary of Strengths and Limitations of CTPA, V/Q SPECT, and V/Q SPECT/CT

\begin{tabular}{|c|c|c|c|}
\hline Parameter & CTPA & V/Q SPECT & V/Q SPECT/CT \\
\hline Sensitivity & Moderate-high & High & High \\
\hline Specificity & Very high & High & Very high \\
\hline Accuracy with abnormal radiograph finding & Unaffected & Sometimes affected & Sometimes affected \\
\hline Ability to provide other diagnoses & Frequent & Rare & Frequent \\
\hline Incidental findings requiring follow-up & Frequent & Rare & Less frequent \\
\hline Radiation dose & High & Low & Low-moderate \\
\hline Possible allergic reaction & Yes & No & No \\
\hline Risk of contrast nephropathy & Yes & No & No \\
\hline Technical failure rate & Higher & Rare & Rare \\
\hline Availability (especially outside routine hours) & High & Usually lower & Usually lower \\
\hline Accuracy in pregnancy & Lower & High & High \\
\hline Accuracy in chronic PE & Lower & High & High \\
\hline Performance in obstructive lung disease & Unaffected & May be affected & May be affected \\
\hline Role and accuracy in follow-up & Limited & Very good & Very good \\
\hline
\end{tabular}

breast from CTPA has been estimated at between 10 and 70 $\mathrm{mSv}$, a particular concern in younger women $(44,45)$. By comparison, the breast radiation dose from the V/Q scan is on the order of $0.3-1 \mathrm{mSv}$ (46). CTPA has overall radiation doses on the order of $8-20 \mathrm{mSv}$, compared with approximately $2.5 \mathrm{mSv}$ with V/Q SPECT (26), also making CTPA unsuitable for follow-up studies to monitor resolution of PE. Finally, there are some concerns related to the detection of incidental or unrelated findings. Although CTPA may diagnose alternate conditions in many patients (up to $33 \%$ in one series), these may not be the cause of patient symptoms (47). Investigation of these incidental findings can be expensive and results in additional radiation or contrast exposure and performance of invasive procedures for uncertain return (48). One study showed that only $3.2 \%$ of CTPA studies in patients with a low or intermediate pretest probability had a relevant alternate diagnosis that was not evident on the chest radiograph (49).

Overall, relatively few studies have directly compared SPECT V/Q and CTPA. Reinartz et al. showed that SPECT was more sensitive (97\% vs. $86 \%$ ) but less specific (91\% vs. $98 \%$ ) than $4-$ slice CTPA (14). Miles et al., in a study of 100 patients using 16-slice CTPA, also found the accuracy of each to be comparable. They noted that SPECT had fewer contraindications,

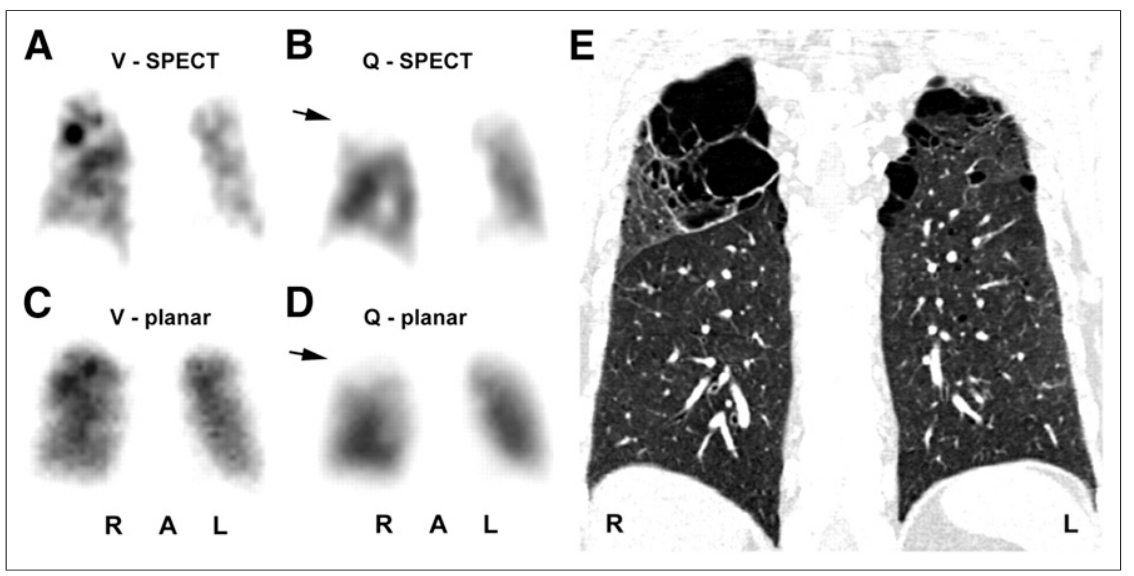

FIGURE 2. (A-D) False-positive V/Q scan due to emphysema. Mismatch is evident in right upper lobe (arrows), but CT (E) shows cause to be emphysematous bulla. $R=$ right; $A=$ anterior; $\mathrm{L}=$ left. (Reprinted with permission from (14).) a lower patient radiation dose, and fewer nondiagnostic findings (50). In a study of 81 patients, Gutte et al. found that V/Q SPECT had a higher sensitivity (97\% compared with 68\%) but a lower specificity ( $88 \%$ compared with $100 \%$ ) than CTPA (16-slice) (18).

These head-to-head studies consistently demonstrate that SPECT has a higher sensitivity, that CTPA has a higher specificity, and that the overall accuracy of each modality is comparable. With each modality having its strengths and weaknesses (Table 2), the test selected for any individual patient should take into account patient factors (including age, sex, renal function, diabetes, and the presence of coexisting lung disease) and institutional factors (e.g., availability and local expertise).

\section{V/Q SPECT/CT}

Although V/Q mismatch is the hallmark of PE, other conditions can cause this appearance (17). Furthermore, not all patients with PE have the classic V/Q mismatch pattern because some develop pulmonary infarction, resulting in matched defects (17). For these reasons, the chest radiograph appearances have been considered pivotal by many to aid in the interpretation of the V/Q scan, and the findings are often used to improve the accuracy and specificity of V/Q reporting $(17,36,51)$.

However, with the development of SPECT/CT scanners, the integration of anatomic information from the CT scan with the functional information from the SPECT scan is now feasible. By combining a V/Q SPECT study with a CT study, even one using "low-dose" settings to reduce patient radiation dose, the advantages of each modality can be realized. V/Q mismatch due to conditions other than PE (such as radiation therapyinduced changes, emphysema, and extrinsic vascular compression from conditions such as neoplasm or mediastinal adenopathy) can be detected by SPECT/CT imaging (Fig. 2) (17). Furthermore, SPECT/ 


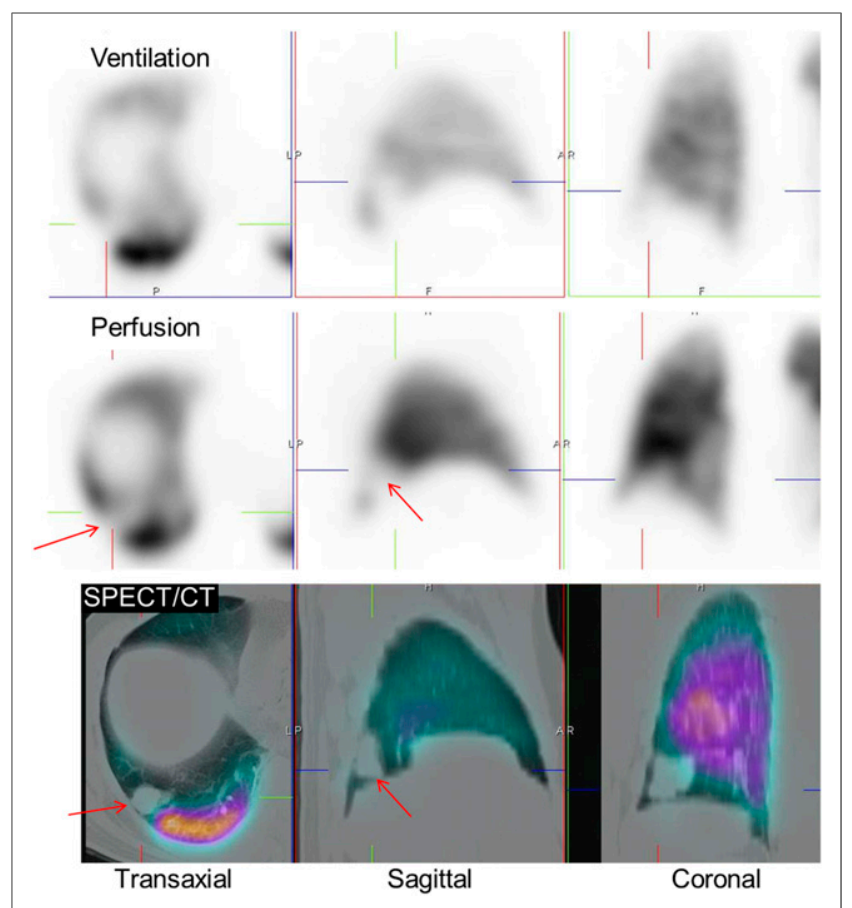

FIGURE 3. Representative SPECT/CT images in patient with coIon cancer and dyspnea. SPECT shows matched defect in right lower lobe (arrows). CT shows this finding to correspond to previously undiagnosed metastasis.

CT can help to characterize matched changes due to nonembolic etiologies such as pneumonia, abscess, pleural or pericardial effusions, malignancy, and pulmonary infarction (Fig. 3) $(3,9,47)$. Hybrid SPECT/CT imaging therefore has the potential to increase the specificity of V/Q scanning by characterizing the causes of underlying perfusion defects $(18,52)$. V/Q SPECT/CT offers the potential for a single imaging procedure yielding a high sensitivity and specificity for the detection of PE that has the added benefit of being able to identify various other conditions that can account for chest pain and dyspnea.

\section{Protocol, Processing, Display, and Reporting}

Dual-detector hybrid SPECT/CT $\gamma$-cameras are now operational in many nuclear medicine departments. Although most devices can be used for diagnostic-quality CT, they can also be operated solely for attenuation correction and anatomic localization using "low-dose" parameters (52).

For lung scanning, the CT acquisition is typically obtained immediately after the perfusion SPECT acquisition. Intravenous contrast material is not required, and a reduced beam current, typically on the order of $20-80 \mathrm{~mA}$, will suffice. The resulting radiation dose is on the order of $1-2 \mathrm{mSv}(18,26)$, comparing favorably with the $2-2.5 \mathrm{mSv}$ from the V/Q scan itself and well below the levels received from diagnostic CTPA $(26,45,53)$. The CT acquisition is rapid ( $<1 \mathrm{~min})$ and, combined with the set-up time, adds only a couple of minutes to a V/Q SPECT study. Although typically used for diagnostic CT studies, breath-holding is not feasible with V/Q SPECT because of the prolonged SPECT acquisitions. To reduce respiratory-motion misregistration, it has been recommended that $\mathrm{CT}$ scans be acquired during breath-holding at mid-inspiration volume, or with the patient continuing shallow breathing during the CT acquisition (54).
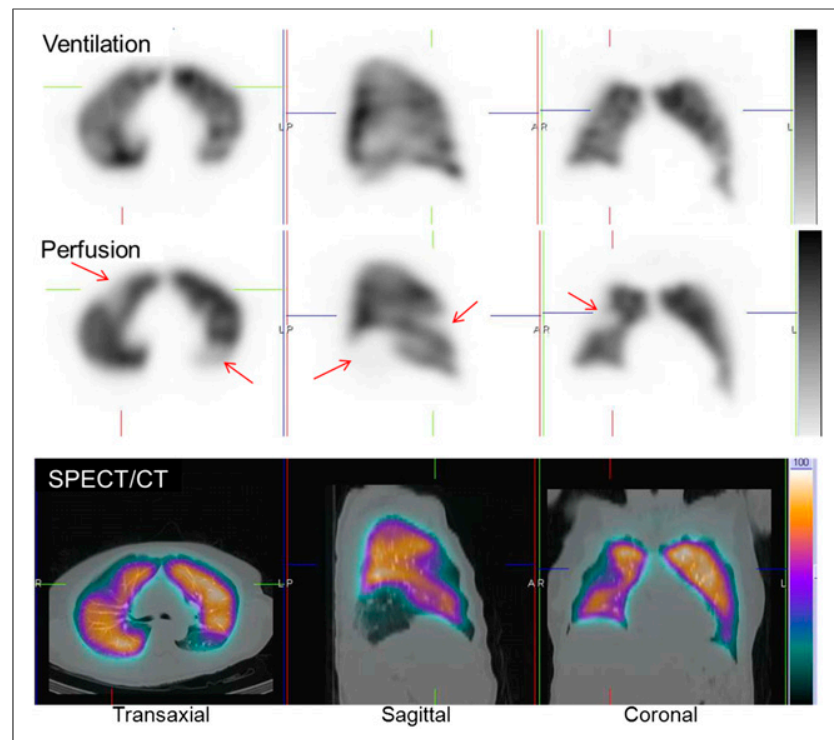

FIGURE 4. Representative SPECT/CT images in patient with multiple PE. Several mismatched defects are evident (arrows). CT shows no underlying structural abnormalities.

As with V/Q SPECT, the European Association of Nuclear Medicine guidelines are recommended for reporting V/Q SPECT/CT studies. Although these guidelines do not specifically address hybrid imaging, the addition of the CT component is likely to help classify the V/Q SPECT pattern more appropriately. The CT scan will provide patient-specific anatomic information, including the lung and segment borders, fissures, and major vessel locations and the presence of any associated parenchymal disease (Fig. 4). The location of the fissures should be noted, as a linear reduction in perfusion (and to a lesser degree ventilation) corresponding to the fissures can be seen on SPECT imaging. The location of any matched changes should also be noted, as consolidative opacities secondary to PE preferentially occur peripherally whereas lesions induced by inflammatory disease tend to be seen at the proximal portion of defects (55).

\section{Clinical Value}

Several studies have shown that combined SPECT/CT lung scanning improves the specificity and overall diagnostic accuracy of lung scintigraphy.

Herald et al. demonstrated a 50\% reduction in false-positive V/Q SPECT studies in a study of 48 patients where SPECT was combined with a low-dose (30-50 mAs) CT scan (56).

A larger prospective study from Gutte et al. demonstrated high diagnostic accuracy when V/Q SPECT was combined with low-dose CT (18). In a series of 81 consecutive patients with ${ }^{81 \mathrm{~m}} \mathrm{Kr}$ gas used as the ventilation agent, V/Q SPECT/CT had an identical sensitivity to V/Q SPECT (97\%). However, the addition of low-dose CT imaging demonstrated that mismatched perfusion defects could be attributed to structures such as fissures and to pathologic conditions such as emphysema, pneumonia, atelectasis, and pleural fluid. As a result, the specificity of scintigraphy increased from $88 \%$ to $100 \%$. The inconclusive rate for V/Q SPECT/CT was zero (compared with $5 \%$ for V/Q SPECT alone). To our knowledge, this study has been the only direct comparison of CTPA (16-slice) with V/Q 


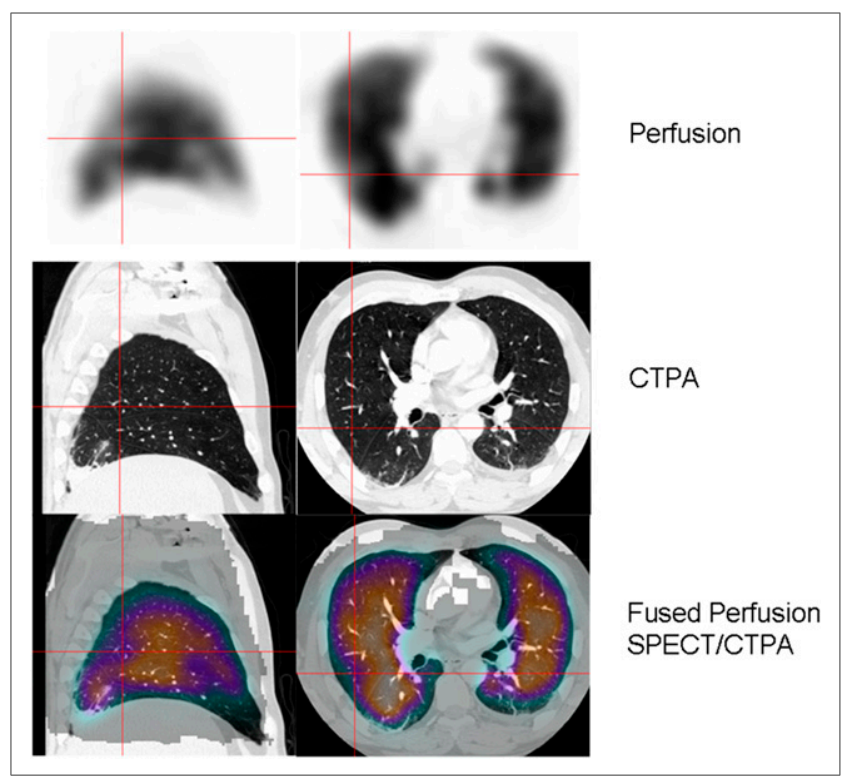

FIGURE 5. Sagittal (left) and transaxial (right) perfusion, CTPA, and fused slices in patient with PE and lower lobe volume loss due to atelectasis. Although defect (red crosshairs) was initially localized to superior segment of right lower lobe, fusion accurately localizes defect to posterior segment of right upper lobe. (Reprinted with permission from (52).)

SPECT/CT (18). Although CTPA had a high specificity ( $100 \%$, identical to that reported for V/Q SPECT/CT), it had a sensitivity much lower than either SPECT or SPECT/CT $(68 \%$ compared with 97\%).

Another benefit of SPECT/CT imaging is the ability to more accurately localize perfusion defects to the correct segments in each individual patient. The segmental reference lung maps that are used to guide SPECT reporting may be erroneous because of the distortion of individual anatomy caused by other lung conditions, such as atelectasis and pleural effusions, which often coexist in patients with PE (Fig. 5) (52,57). This information may be relevant to help guide a reporting radiologist to the correct segmental artery should CTPA be required to confirm the findings on a V/Q SPECT study.

\section{Combining V/Q SPECT with CTPA}

Another option for combining structural and functional images is to fuse perfusion SPECT with diagnostic CTPA, performed on either the same hybrid scanner or another CT scanner using software fusion (Fig. 6) (58). Although requiring appropriate software programs and operator expertise, this approach can be of value in

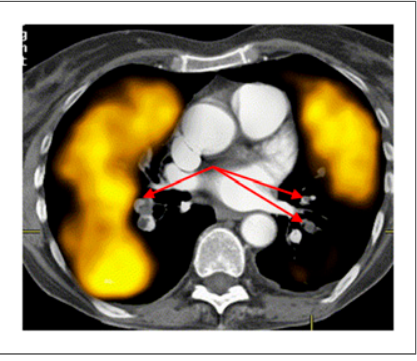

FIGURE 6. Coregistered CTPA and perfusion SPECT scans (transverse slice) demonstrating extensive perfusion defects on SPECT. Findings correspond to proximal bilateral PE shown on CTPA (arrows) (Reprinted with permission from (52).)

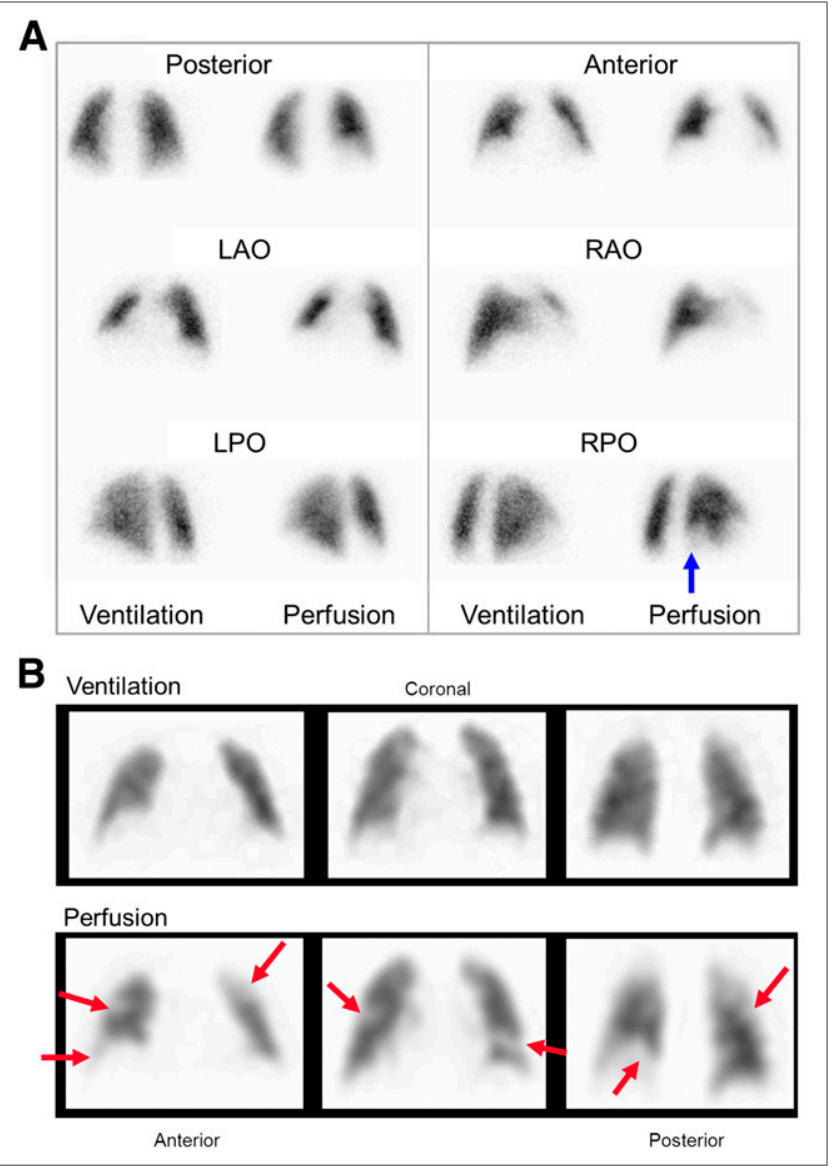

FIGURE 7. (A) Planar V/Q scan in patient with dyspnea. Single mismatched defect is seen at right base (arrow), classifying study as intermediate probability of PE. (B) Representative coronal SPECT slices show multiple mismatched defects (arrows) indicative of widespread PE. Patient had extensive deep venous thrombosis.

selected patients and may better guide the reporting radiologist to the site of a likely clot on CTPA (59).

\section{CONTROVERSIES}

Is the Ventilation Scan Necessary?

With SPECT/CT able to show structural abnormalities, the need for a ventilation study has been questioned. Several studies have demonstrated that specificity falls significantly if ventilation is omitted. Gradinscak et al. showed that parenchymal abnormalities (usually subsegmental atelectasis) were noted on CT in $13 \%$ of V/Q SPECT mismatches (60), and Gutte et al. demonstrated that perfusion-only SPECT/CT has a higher nondiagnostic rate $(17 \%)$ and lower specificity than V/Q SPECT/CT $(51 \%$ compared with $100 \%$ ) (18). Although perfusion-only SPECT/CT should be considered in sites without access to a suitable ventilation agent, limited literature suggests that performing a ventilation study does maximize specificity and reduce false-positive results.

\section{Do Additional Clots Detected by SPECT Warrant Treatment?}

Although V/Q SPECT (and SPECT/CT) has a higher sensitivity than planar imaging, the question has been raised as to 

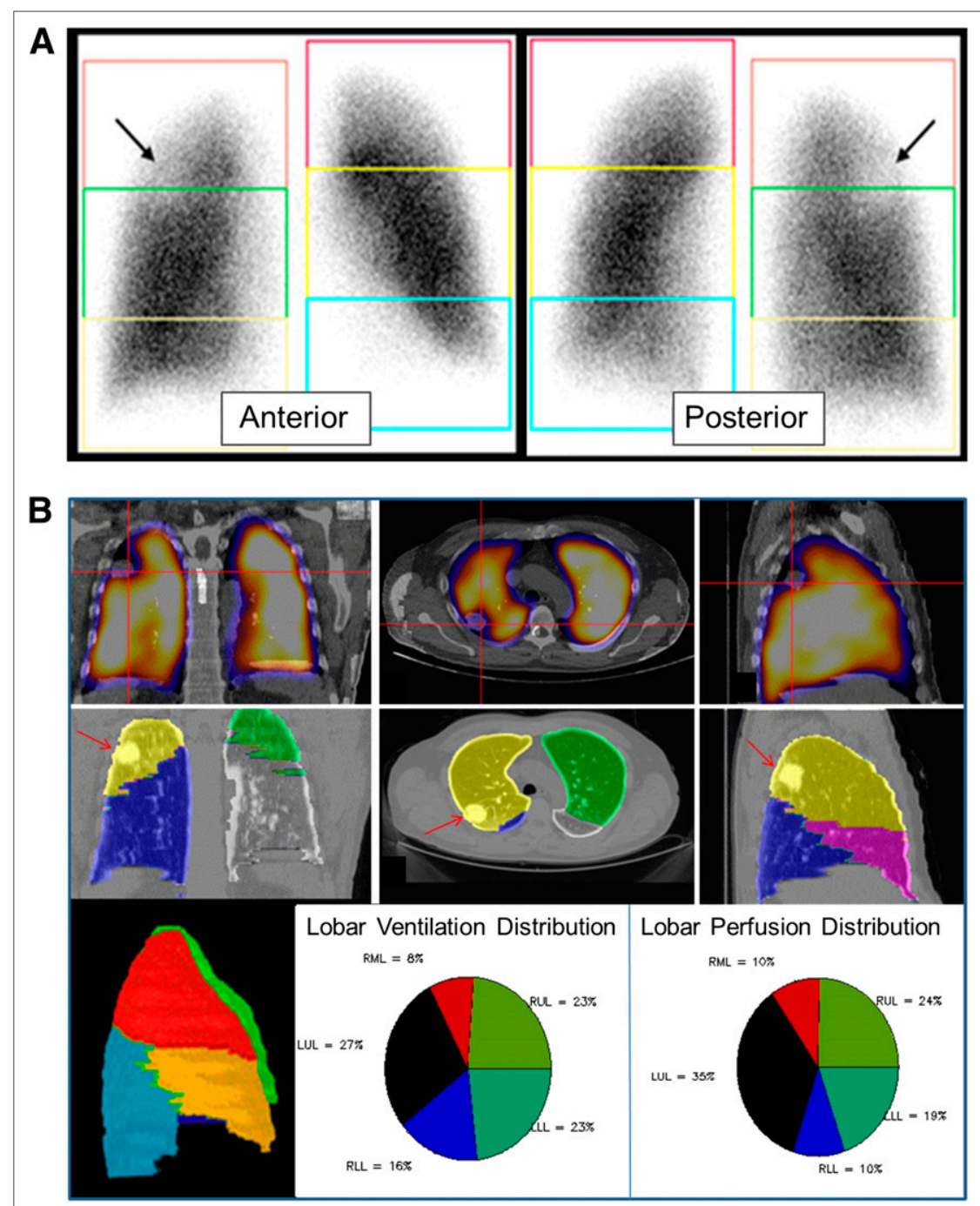

FIGURE 8. (A) Anterior (left) and posterior (right) planar images in patient with right lung carcinoma (arrows). Boxes over upper, middle, and lower thirds of each lung approximate relative contribution of each region. Because of overlap of segments and differences in individual anatomy, accuracy is lacking. (B) Fused perfusion/CT images (top row) in coronal (left), transverse (middle), and sagittal (right) planes show perfusion defect (due to tumor, denoted with red crosshairs) in right upper lobe. Patient's individual CT scan can be used to generate patient-specific lobar slices (middle row) in corresponding orthogonal slices and rotating maximum-intensity-projection images (left image, bottom row). SPECT/ CT allowed accurate determination of each lobe's relative contribution to overall ventilation (middle image, bottom row) and perfusion (right image, bottom row). LLL = left lower lobe; $\mathrm{LUL}=$ left upper lobe; $\mathrm{RLL}=$ right lower lobe; $\mathrm{RML}=$ right middle lobe; $\mathrm{RUL}=$ right upper lobe.

whether these clots (which are often subsegmental) are significant enough to warrant anticoagulation $(3,23,53,61)$. Although large prospective outcome studies would be needed to answer this question (and would be welcomed), it is our experience (and that of others) that SPECT detects additional PE not just at the subsegmental level (Fig. 7) (14). Diagnosis of any PE, including small ones, may be of particular importance in patients with impaired cardiopulmonary reserve, coexisting DVT, or recurrent small PE (with its risk of pulmonary hypertension) (62). Although this consideration raises the broader philosophic question as to whether diagnostic accuracy or clinical outcome is more relevant, we consider that the higher sensitivity and accuracy, improved reader confidence, greater ease of reporting, and ability to perform hybrid SPECT/CT imaging are all compelling reasons to replace planar imaging with SPECT for imaging PE.

\section{NON-PE APPLICATIONS AND FUTURE DIRECTIONS}

V/Q SPECT and SPECT/CT have utility in areas other than PE. In patients undergoing lung volume reduction surgery, SPECT/CT can provide a more accurate assessment of relative perfusion (and ventilation) of individual segments than planar imaging (Fig. 8). V/Q SPECT has been shown to have use in areas such as predicting postoperative lung function after lung resection in patients with lung cancer, modifying radiotherapy fields to minimize radiation exposure to functioning lung, demonstrating regional changes of ventilation and perfusion in asthma, and estimating regional lung function in patients with interstitial pulmonary disease (63-65).

Future directions for SPECT/CT are likely to include radiolabeled thrombus imaging of PE (66). Quantitative SPECT will benefit from hybrid imaging through derivation of accurate patient-specific attenuation correction (34). Lung scanning using PET radiopharmaceuticals, such as ${ }^{68}$ Ga-carbon nanoparticles ("Galligas") and ${ }^{68} \mathrm{Ga}$-macroaggregated albumin, is a promising development that may further enhance the role of nuclear medicine in the assessment of PE and other lung disorders (49).

\section{CONCLUSION}

As with other areas of nuclear medicine, V/Q scanning has been improved significantly with SPECT imaging. V/Q SPECT has a higher sensitivity and specificity than planar imaging, as well as reduced interobserver variability, increased overall accuracy, and improved reporter confidence. The low rate of technically adequate studies, absence of contrast-related risks, and higher sensitivity and lower radiation dose than CTPA are all arguments supporting the use of V/Q SPECT as the initial screening test for PE in most patients $(33,41)$. Specificity is further enhanced by V/Q SPECT/CT, which provides the potential for a single imaging procedure to assess PE with high accuracy while also excluding various alternative causes of chest pain and dyspnea. 


\section{REFERENCES}

1. Wagner HN Jr, Sabiston DC Jr, McAfee JG, Tow D, Stern HS. Diagnosis of massive pulmonary embolism in man by radioisotope scanning. $N$ Engl J Med. 1964;271:377-384.

2. Meignan MA. Lung ventilation/perfusion SPECT: the right technique for hard times. J Nucl Med. 2002;43:648-651.

3. Anderson DR, Kahn SR, Rodger MA, et al. Computed tomographic pulmonary angiography vs ventilation-perfusion lung scanning in patients with suspected pulmonary embolism: a randomized controlled trial. JAMA. 2007;298: 2743-2753.

4. Morrell NW, Nijran KS, Jones BE, Biggs T, Seed WA. The underestimation of segmental defect size in radionuclide lung scanning. J Nucl Med. 1993;34:370374 .

5. Magnussen JS, Chicco P, Palmer AW, et al. Single-photon emission tomography of a computerised model of pulmonary embolism. Eur J Nucl Med. 1999;26: 1430-1438.

6. Goodman LR, Lipchik RJ. Diagnosis of acute pulmonary embolism: time for a new approach. Radiology. 1996;199:25-27.

7. Gray HW, McKillop JH, Bessent RG. Lung scan reporting language: what does it mean? Nucl Med Commun. 1993;14:1084-1087.

8. Stein PD, Freeman LM, Sostman HD, et al. SPECT in acute pulmonary embolism. J Nucl Med. 2009;50:1999-2007.

9. Strashun AM. A reduced role of V/Q scintigraphy in the diagnosis of acute pulmonary embolism. J Nucl Med. 2007;48:1405-1407.

10. Osborne DR, Jaszczak RJ, Greer K, Roggli V, Lischko M, Coleman RE. Detection of pulmonary emboli in dogs: comparison of single photon emission computed tomography, gamma camera imaging, and angiography. Radiology. 1983;146:493-497.

11. Bajc M, Bitzen U, Olsson B, Perez de Sa V, Palmer J, Jonson B. Lung ventilation/perfusion SPECT in the artificially embolized pig. J Nucl Med. 2002;43:640-647.

12. Bajc M, Olsson CG, Olsson B, Palmer J, Jonson B. Diagnostic evaluation of planar and tomographic ventilation/perfusion lung images in patients with suspected pulmonary emboli. Clin Physiol Funct Imaging. 2004;24:249-256.

13. Collart JP, Roelants V, Vanpee D, et al. Is a lung perfusion scan obtained by using single photon emission computed tomography able to improve the radionuclide diagnosis of pulmonary embolism? Nucl Med Commun. 2002; 23:1107-1113.

14. Reinartz P, Wildberger JE, Schaefer W, Nowak B, Mahnken AH, Buell U. Tomographic imaging in the diagnosis of pulmonary embolism: a comparison between V/Q lung scintigraphy in SPECT technique and multislice spiral CT. J Nucl Med. 2004;45:1501-1508.

15. Bajc M, Olsson B, Palmer J, Jonson B. Ventilation/perfusion SPECT for diagnostics of pulmonary embolism in clinical practice. J Intern Med. 2008; 264:379-387.

16. Lemb M, Pohlabeln H. Pulmonary thromboembolism: a retrospective study on the examination of 991 patients by ventilation/perfusion SPECT using Technegas. Nuklearmedizin. 2001;40:179-186.

17. Bajc M, Neilly JB, Miniati M, et al. EANM guidelines for ventilation/ perfusion scintigraphy: part 1 . Pulmonary imaging with ventilation/perfusion single photon emission tomography. Eur J Nucl Med Mol Imaging. 2009;36:1356-1370

18. Gutte H, Mortensen J, Jensen CV, et al. Detection of pulmonary embolism with combined ventilation-perfusion SPECT and low-dose CT: head-to-head comparison with multidetector CT angiography. J Nucl Med. 2009;50:1987-1992.

19. Roach PJ, Bailey DL, Harris BE. Enhancing lung scintigraphy with singlephoton emission computed tomography. Semin Nucl Med. 2008;38:441-449.

20. Leblanc M, Leveillee F, Turcotte E. Prospective evaluation of the negative predictive value of V/Q SPECT using ${ }^{99 \mathrm{~m}}$ Tc-Technegas. Nucl Med Commun. 2007;28:667-672.

21. Petersson J, Sanchez-Crespo A, Larsson SA, Mure M. Physiological imaging of the lung: single-photon-emission computed tomography (SPECT). J Appl Physiol. 2007;102:468-476.

22. Senden TJ, Moock KH, Gerald JF, et al. The physical and chemical nature of Technegas. J Nucl Med. 1997;38:1327-1333.

23. Freeman LM, Glaser JE, Haramati LB. Planar ventilation-perfusion imaging for pulmonary embolism: the case for "outcomes" medicine. Semin Nucl Med. 2012;42:3-10.

24. Jögi J, Jonson B, Ekberg M, Bajc M. Ventilation-perfusion SPECT with ${ }^{99 \mathrm{~m}} \mathrm{Tc}$ DTPA versus Technegas: a head-to-head study in obstructive and nonobstructive disease. J Nucl Med. 2010;51:735-741.

25. Bailey EA, Bailey DL, Roach PJ. V/Q imaging in 2010: a quick start guide. Semin Nucl Med. 2010;40:408-414.
26. Schembri GP, Miller AE, Smart R. Radiation dosimetry and safety issues in the investigation of pulmonary embolism. Semin Nucl Med. 2010;40: 442-454.

27. Roach PJ, Bailey DL, Schembri GP, Thomas PA. Transition from planar to SPECT V/Q scintigraphy: rationale, practicalities, and challenges. Semin Nucl Med. 2010;40:397-407.

28. Bailey DL, Schembri GP, Harris BE, Bailey EA, Cooper RA, Roach PJ. Generation of planar images from lung ventilation/perfusion SPECT. Ann Nucl Med. 2008;22:437-445.

29. Harris B, Bailey DL, Roach PJ, et al. A clinical comparison between traditional planar V/Q images and planar images generated from SPECT V/Q scintigraphy. Nucl Med Commun. 2008;29:323-330.

30. Palmer J, Bitzen U, Jonson B, Bajc M. Comprehensive ventilation/perfusion SPECT. J Nucl Med. 2001;42:1288-1294.

31. Harris B, Bailey D, Miles S, et al. Objective analysis of tomographic ventilationperfusion scintigraphy in pulmonary embolism. Am J Respir Crit Care Med. 2007; 175:1173-1180.

32. Harris B, Bailey DL, Chicco P, Bailey EA, Roach PJ, King GG. Objective analysis of whole lung and lobar ventilation/perfusion relationships in pulmonary embolism. Clin Physiol Funct Imaging. 2008; 28:14-26.

33. Roach PJ, Bailey DL, Schembri GP. Reinventing ventilation/perfusion lung scanning with SPECT. Nucl Med Commun. 2008;29:1023-1025.

34. Willowson K, Bailey DL, Bailey EA, Baldock C, Roach PJ. In vivo validation of quantitative SPECT in the heart. Clin Physiol Funct Imaging. 2010;30: 214-219.

35. Stein PD, Fowler SE, Goodman LR, et al. Multidetector computed tomography for acute pulmonary embolism. N Engl J Med. 2006;354:23172327.

36. Value of the ventilation/perfusion scan in acute pulmonary embolism: results of the prospective investigation of pulmonary embolism diagnosis (PIOPED). The PIOPED Investigators. JAMA. 1990;263:2753-2759.

37. Jones SE, Wittram C. The indeterminate CT pulmonary angiogram: imaging characteristics and patient clinical outcome. Radiology. 2005;237:329337.

38. U-King-Im JM, Freeman SJ, Boylan T, Cheow HK. Quality of CT pulmonary angiography for suspected pulmonary embolus in pregnancy. Eur Radiol. 2008;18:2709-2715.

39. Laurence IJ, Redman SL, Corrigan AJ, Graham RN. V/Q SPECT imaging of acute pulmonary embolus: a practical perspective. Clin Radiol. 2012;67:941948.

40. Ridge CA, McDermott S, Freyne BJ, Brennan DJ, Collins CD, Skehan SJ. Pulmonary embolism in pregnancy: comparison of pulmonary CT angiography and lung scintigraphy. AJR. 2009;193:1223-1227.

41. Leblanc M, Paul N. V/Q SPECT and computed tomographic pulmonary angiography. Semin Nucl Med. 2010;40:426-441.

42. Toney LK, Lewis DH, Richardson ML. Ventilation/perfusion scanning for acute pulmonary embolism: effect of direct communication on patient treatment outcomes. Clin Nucl Med. 2013;38:183-187.

43. Barrett BJ, Parfrey PS. Clinical practice: preventing nephropathy induced by contrast medium. N Engl J Med. 2006;354:379-386.

44. Parker MS, Hui FK, Camacho MA, Chung JK, Broga DW, Sethi NN. Female breast radiation exposure during CT pulmonary angiography. AJR. 2005;185: 1228-1233.

45. Hurwitz LM, Yoshizumi TT, Goodman PC, et al. Radiation dose savings for adult pulmonary embolus 64-MDCT using bismuth breast shields, lower peak kilovoltage, and automatic tube current modulation. AJR. 2009;192: 244-253.

46. ICRP. Radiation dose to patients from radiopharmaceuticals (addendum 2 to ICRP publication 53). Ann ICRP. 1998;28:1-126.

47. Hall WB, Truitt SG, Scheunemann LP, et al. The prevalence of clinically relevant incidental findings on chest computed tomographic angiograms ordered to diagnose pulmonary embolism. Arch Intern Med. 2009;169:19611965 .

48. Schattner A. Computed tomographic pulmonary angiography to diagnose acute pulmonary embolism: the good, the bad, and the ugly. Arch Intern Med. 2009;169:1966-1968.

49. Chandra S, Sarkar PK, Chandra D, Ginsberg NE, Cohen RI. Finding an alternative diagnosis does not justify increased use of CT-pulmonary angiography. $B M C$ Pulm Med. 2013;13:9.

50. Miles S, Rogers KM, Thomas P, et al. A comparison of single-photon emission CT lung scintigraphy and CT pulmonary angiography for the diagnosis of pulmonary embolism. Chest. 2009;136:1546-1553. 
51. Miniati M, Pistolesi M, Marini C, et al. Value of perfusion lung scan in the diagnosis of pulmonary embolism: results of the Prospective Investigative Study of Acute Pulmonary Embolism Diagnosis (PISA-PED). Am J Respir Crit Care Med. 1996;154:1387-1393.

52. Roach PJ, Gradinscak DJ, Schembri GP, Bailey EA, Willowson KP, Bailey DL. SPECT/CT in V/Q scanning. Semin Nucl Med. 2010;40:455-466.

53. Freeman LM. Don't bury the V/Q scan: it's as good as multidetector CT angiograms with a lot less radiation exposure. J Nucl Med. 2008;49:5-8.

54. Delbeke D, Coleman RE, Guiberteau MJ, et al. Procedure guideline for SPECT/ CT imaging 1.0. J Nucl Med. 2006;47:1227-1234.

55. Zaki M, Suga K, Kawakami Y, et al. Preferential location of acute pulmonary thromboembolism induced consolidative opacities: assessment with respiratory gated perfusion SPECT-CT fusion images. Nucl Med Commun. 2005;26:465474.

56. Herald P, Roach P, Schembri GP. Does the addition of low dose CT improve diagnostic accuracy of V/Q SPECT scintigraphy? [abstract]. J Nucl Med. 2008;49(suppl):91P.

57. Gradinscak DJ, Roach P, Schembri GP. Can CT coregistration improve the accuracy of segmental localisation on V/Q SPECT? [abstract]. Eur J Nucl Med Mol Imaging. 2009;36(suppl):S463.

58. Harris B, Bailey D, Roach P, Bailey E, King G. Fusion imaging of computed tomographic pulmonary angiography and SPECT ventilation/perfusion scintig- raphy: initial experience and potential benefit. Eur J Nucl Med Mol Imaging. 2007;34:135-142.

59. Gradinscak DJ, Roach P, Schembri GP. Can perfusion SPECT improve the accuracy of CTPA? [abstract]. Eur J Nucl Med Mol Imaging. 2009;36(suppl): S463.

60. Gradinscak DJ, Roach P, Schembri GP. Lung SPECT perfusion scintigraphy: can CT substitute for ventilation imaging? [abstract]. Eur J Nucl Med Mol Imaging. 2009;36(suppl):S300.

61. Freeman LM, Haramati LB. V/Q scintigraphy: alive, well and equal to the challenge of CT angiography. Eur J Nucl Med Mol Imaging. 2009;36:499-504.

62. Goodman LR. Small pulmonary emboli: what do we know? Radiology. 2005;234:654-658.

63. King GG, Harris B, Mahadev S. V/Q SPECT: utility for investigation of pulmonary physiology. Semin Nucl Med. 2010;40:467-473.

64. Christian JA, Partridge M, Nioutsikou E, et al. The incorporation of SPECT functional lung imaging into inverse radiotherapy planning for non-small cell lung cancer. Radiother Oncol. 2005;77:271-277.

65. Pellegrino R, Biggi A, Papaleo A, Camuzzini G, Rodarte JR, Brusasco V. Regional expiratory flow limitation studied with Technegas in asthma. J Appl Physiol. 2001;91:2190-2198.

66. Morris TA. SPECT imaging of pulmonary emboli with radiolabeled thrombusspecific imaging agents. Semin Nucl Med. 2010;40:474-479. 